\title{
EDITORIAL
}

\section{SHOULD NURSING ETHICS BE DISTINGUISHED FROM MEDICAL ETHICS?}

Medical care is much boarder than simply restoring normal function. It is the interaction between care and cure characterizing by care and trust, constitute the essence of doctor patient relationship and nurse-patient relationship. A book entitled "Extending the boundary of care: medical ethics and caring practice" introduce the limit between the care and cure.

Medical ethics exclusively focus on medical decision where care is relatively neglected. Medical decision of care is taken by nurse. In a nursing home dimension, care for older person, person suffering from chronic condition, mentally incorporated older person, dying patients etc are responsible for nurse. In curative medical care, care for these people is limited. For them role of nursing care is more important. Nurse usually confronts ethical problem arise in their practice. So this care needs different type of ethics.

On the basis of philosophical analysis, concept of care is the reflection of value of nursing profession. Ethics of care is emphasis on responsibility, solidarity, finitude, morality and communication. It is long tradition nurse has been demonstrating the value of ethics of care. In addition, nursing profession is largely feminine, care oriented, virtue and emotional then the medical ethics which is masculine, cure oriented including principal and rational. Therefore there is room for thinking to distinguish nursing ethics from medical ethics. Research on this arena is needed.

Shamima Parvin Lasker

(Professor \& Head of Anatomy, City Dental College, Bangladesh)

Associate Editor

Bangladesh Journal of Bioethics 